\title{
Sulle \\ curve multiple di superficie algebriche
}

\author{
(del Dr. Max Noether, a Heidelberg.)
}

\begin{abstract}
Qui mi propongo di riunire alcune formole relative al sistema dei punti d'intersezione di tre superficie, che abbiano in comune una curva multipla, ed anche relative al numero delle condizioni che per una superficie sono assorbite da tali curve. Questi risultati sono qui presentati soltanto come estensioni 0 anche come esatte determinazioni di altri risultati già noti, dovuti principalmente al sig. CAYLEY (*); ma appunto per la precisione della loro forma, sembrano ora suscettivi di molteplici applicazioni a diversi rami della geometria. Alla fine di questi scritti, si troverà l'applicazione alle trasformazioni birazionali (eindeutig) nello spazio.
\end{abstract}

\section{L' equivalenza di una curva multipla.}

1. Nella Geometry of three dimensions di SALMON (**) si trova l'espressione del numero de'punti d'intersezione di tre superficie, assorbiti da una comune curva, che sia semplice per due superficie e doppia per la terza. Adoperando semplicemente, come già ha fatto il sig. C CAYLEY (****), una ripetizione del processo ivi usato, si ottiene gradualmente l'espressione generale del numero di punti assorbiti da una curva, che sia multipla per ciascuna delle tre superficie. Questo numero si dirà l'equivalenza della curva.

(*) Vedi particolarmente le formole di equivalence e di postulation di Cayley nella sua Memoria On the rational transformation between troo spaces (Proceedings of the London math. Society, vol. 3, 1870, p. 179).

(**) Pag. 280. Cfr. anche CRemona, Preliminari n. 97.

(***) On reciprocal surfaces (Phil. Transact. vol. 159, 1869, p. 221). 
2. Sia $m$ l'ordine della curva; $r$ il rango della medesima, vale a dire l'ordine della sviluppabile formata dalle sue tangenti; e la curva abbia $h$ punti doppi apparenti, $k$ punti doppi effettivi; dove per ora intendo di escludere punti di più alta moltiplicità. Allora è

$$
r=m(m-1)-2 h-2 k \text {. }
$$

Le tre superficie $F_{1}, F_{2}, F_{3}$ siano rispettivamente d'ordine $n_{1}, n_{2}, n_{3}$, e per esse la curva $C$ sia multipla secondo $i_{1}, i_{2}, i_{3}$. La curva sarà equivalente a

$$
m\left[i_{2} i_{3} n_{1}+i_{3} i_{1} n_{2}+i_{1} i_{2} n_{3}\right]-2 i_{1} i_{2} i_{3}\left(m+\frac{r+2 k}{2}\right)
$$

punti d'intersezione delle tre superficie. Poi, la curva $G$ incontra la curva residua d'intersezione delle due superficie $F_{1}, F_{2}$ in

punti.

$$
m\left(i_{2} n_{1}+i_{1} n_{2}\right)-2 i_{1} i_{2}\left(m+\frac{r+2 k}{2}\right)
$$

3. Passo oltre a determinare il numero delle intersezioni assorbite da più curve multiple, che fra loro si seghino. A quest' uopo, cerco la riduzione che le equivalenze di due curve subiscono in conseguenza di un punto ad esse comune. Siccome questa riduzione non puó dipendere che dalla configurazione delle due curve in prossimita del punto d'intersezione, così io la dedurro dal caso semplicissimo che le due curve siano due rette segantisi $G, G^{\prime}$.

La retta $G$ sia multipla secondo $i_{1}, i_{2}, i_{3}$ per le tre superficie $F_{1}, F_{2}, F_{3}$; e $i_{1}^{\prime}, i_{2}^{\prime}, i_{3}^{\prime}$ siano i numeri analoghi per $G^{\prime}$; e suppongasi $i_{1} \geqq i_{1}^{\prime}, i_{2} \geqq i_{2}^{\prime}$.

La curva residua d'intersezione delle due superficie $F_{1}, F_{2}$ ha allora con $G$

e $\operatorname{con} G^{\prime}$

$$
i_{2} n_{1}+i_{1} n_{2}-2 i_{1} i_{2}-i_{1}^{\prime} i_{2}^{\prime}
$$

$$
i_{2}^{\prime} n_{1}+i_{1}^{\prime} n_{2}-2 i_{1}^{\prime} i_{2}^{\prime}-\left(i_{1} i_{2}^{\prime}+i_{2} i_{1}^{\prime}-i_{1}^{\prime} i_{2}^{\prime}\right)
$$

punti comuni e non passa più oltre pel punto $G G^{\prime}$. Dall' intersezione di questa curva con $F_{3}$ segue pertanto che l'equivalenza del sistema delle due rette è

$$
\begin{aligned}
& \left(i_{2} i_{3} n_{1}+i_{3} i_{1} n_{2}+i_{1} i_{2} n_{3}\right)-2 i_{1} i_{2} i_{3}+ \\
+ & \left(i_{2}^{\prime} i_{3}^{\prime} n_{1}+i_{3}^{\prime} i_{1}^{\prime} n_{2}+i_{1}^{\prime} i_{2}^{\prime} n_{3}\right)-2 i_{1}^{\prime} i_{2}^{\prime} i_{3}^{\prime}- \\
- & \left(i_{2}^{\prime} i_{3}^{\prime} i_{1}+i_{3}^{\prime} i_{1}^{\prime} i_{2}{ }^{\prime} \cdot i_{1}^{\prime} i_{2}^{\prime} i_{3}-i_{1}^{\prime} i_{2}^{\prime} i_{3}^{\prime}\right) .
\end{aligned}
$$


4. Le superficie $F_{1}, F_{2}, F_{3}$ abbiano ora in comune due curve $C$ e $C^{\prime}$, la prima delle quali sia multipla secondo $i$ numeri $i_{1}, i_{2}, i_{3}$, e l'altra secondo i numeri $i_{1}^{\prime}, i_{2}^{\prime}, i_{3}^{\prime}$. Le equivalenze di queste due curve siano $M, M^{\prime}$. Se $C$ e $C^{\prime}$ si segano in $s$ punti, l'equivalenza del loro sistema sarà

$$
M+M^{\prime}-s\left(i_{2}{ }^{\prime} i_{3}{ }^{\prime} i_{1}+i_{3}{ }^{\prime} i_{1}{ }^{\prime} i_{2}+i_{1}^{\prime} i_{2}{ }^{\prime} i_{3}-i_{1}{ }^{\prime} i_{2}^{\prime} i_{3}^{\prime}\right) \text {, }
$$

dove si supponga che due dei numeri $i_{1}, i_{2}, i_{3}$ siano uguali o maggiori dei corrispondenti numeri $i_{1}^{\prime}, i_{2}^{\prime}, i_{s}^{\prime}$.

E la curva residua d'intersezione di $F_{1}, F_{2}$ incontra $C$, ossia la curva $(m, r, k)$, in

$$
\left.\left[m\left(i_{2} n_{1}+i_{1} n_{2}\right)-2 i_{1} i_{2}\left(m+\frac{r+2 k}{2}\right)\right]-i_{1}^{\prime} i_{2}^{\prime} s \text { (per } i_{\mathrm{t}} \geqq i_{1}^{\prime}, i_{2} \gtreqless i_{2}^{\prime}\right),
$$

punti; incontra invece $C^{\prime}$, ossia la curva $\left(m^{\prime}, r^{\prime}, k^{\prime}\right)$, in

$$
\left[m^{\prime}\left(i_{2}^{\prime} n_{1}+i_{1}^{\prime} n_{2}\right)-2 i_{1}^{\prime} i_{2}^{\prime}\left(m^{\prime}+\frac{r^{\prime}+2 k^{\prime}}{2}\right)\right]-\left(i_{1} i_{2}{ }^{\prime}+i_{2} i_{1}^{\prime}-i_{1}{ }^{\prime} i_{2}{ }^{\prime}\right) s
$$

punti, se $i_{1} \geqq i_{1}^{\prime}, i_{2} \geqq i_{2}^{\prime}$, ed in

$$
\left[m^{\prime}\left(i_{2}^{\prime} n_{1}+i_{1}^{\prime} n_{2}\right)-2 i_{1}^{\prime} i_{2}^{\prime}\left(m^{\prime}+\frac{r^{\prime}+2 k^{\prime}}{2}\right)\right]-i_{1} i_{2} s
$$

punti, se $i_{1} \geqq i_{1}^{\prime}, i_{2} \leqq i_{2}^{\prime}$.

Qui osservo che la riduzione ora ottenuta dell'equivalenza $M+M^{\prime}$ sussiste anche pei punti doppi effettivi di una curva, perchè il termine della formola al n. ${ }^{\circ} 2$, che contjene il fattore $k$, si può riguardare come esprimente la riduzione dovuta a tali punti; il che accadrà anche in seguito.

5. Trattisi ora il seguente caso generale.

Le superficie $F_{1}, F_{2}, F_{3}$ abbiano il punto comune $P$, multiplo rispettivamente secondo i numeri $l_{1}, l_{2}, l_{3}$, e le curve comuni $C, C^{\prime}, \ldots$, delle quali la prima sia contenuta $i_{1}, i_{2}, i_{3}$ volte e passi con $j$ rami per $P$, la seconda sia contenuta $i_{1}{ }^{\prime}, i_{2}{ }^{\prime}, i_{3}{ }^{\prime}$ volte ed abbia $j^{\prime}$ rami passanti per $P$, ecc. Suppongasi poi che i coni osculatori alle superficie $F$ in $P$ non si decompongano, in virtù delle singolarità ammesse, in parti che siano comuni ai coni medesimi: il che involge un limite inferiore per le $l_{1}, l_{2}, l_{3}$.

La deduzione dell'equivalenza di un siffatto sistema puo attuarsi ancora col metodo indicato nel n. ${ }^{\circ}$ 3. A ciascun ramo delle curve $C, C^{\prime}, \ldots$ si sostituiscano rette dotate della corrispondente moltiplicità e uscenti da $P$; e si determini direttamente la riduzione dell'equivalenza di questo nuovo sistema. Io daró a dirittura il risultato della deduzione. 
Il rango $r$ di $C$ è nel caso attuale

$$
r=m(m-1)-2 h-2 k-j(j-1)
$$

e come equivalenza $M$ di $C$ s'intenda la quantità

$$
M=m\left(i_{2} i_{3} n_{1}+i_{3} i_{1} n_{2}+i_{1} i_{2} n_{3}\right)-2 i_{1} i_{2} i_{3}\left(m+\frac{r}{2}\right) .
$$

Analogo significato abbia $M^{\prime}$ per $C^{\prime}$, ecc. L'equivalenza

$$
l_{1} l_{2} l_{3}+M+M^{\prime}+\cdots
$$

del sistema $\left(P, G, C^{\prime}, \ldots\right)$ subisce la riduzione

$$
-\sum_{\rho}\left\{i_{2}{ }^{(\rho)} i_{3}{ }^{(\rho)} l_{1}+i_{3}{ }^{(\rho)} i_{1}{ }^{(\varrho)} l_{2}+i_{1}{ }^{(\varrho)} i_{2}{ }^{(\rho)} l_{3}-2 i_{1}{ }^{(\rho)} i_{2}{ }^{(\rho)} i_{3}{ }^{(\rho)}\right\} j^{(\rho)}
$$

dove la somma s'intende estesa alle diverse curve passanti per $P$.

La curva residua d'intersezione delle superficie $F_{1}, F_{2}$ incontra la curva $C$ in

$$
\left[m\left(i_{2} n_{1}+i_{1} n_{2}\right)-2 i_{1} i_{2}\left(m+\frac{r+2 k}{2}\right)\right]-\left(i_{2} l_{1}+i_{1} l_{2}-2 i_{1} i_{2}\right) j
$$

punti, oltre a $P$, e passa con

rami pel punto $P$.

$$
l_{1} l_{2}-\sum_{\rho} i_{1}{ }^{(\varrho)} i_{2}{ }^{(\varrho)} j(\varrho)
$$

6. Se l'ipotesi fatta nel $n .^{\circ} 5$ non è soddisfatta, come p. es. nel caso del $n .^{\circ} 4$, tuttavia si raggiunge ancora lo scopo mediante considerazioni analoghe a quelle fatte nel $n .^{\circ} 3$. Siccome la grande moltiplicità de' casi particolari non permette di giungere ad una formola generale, cosi io mi limito a riferire il seguente esempio.

Le tre superficie $F$ siano d'ordine $n$ e posseggano tre rette multiple secondo $i$ e uscenti da un punto $P$. Il punto $P$ deve allora essere multiplo per le $F$ secondo $\frac{3 i}{2} 0$ secondo $\frac{3 i+1}{2}$, secondochè $i$ è pari o dispari. Se $n$ è abbastanza grande, onde le $F$ non abbiano a decomporsi, l'equivalenza del sistema sarà per $i$ pari

per $i$ dispari

$$
\left(9 n i^{2}-6 i^{3}\right)-\frac{9}{2} i^{3}
$$

$$
\left(9 n i^{2}-6 i^{3}\right)-\left(\frac{9}{2} i^{3}-\frac{1}{2}\right),
$$

e la curva residua d'intersezione di due $F$ nel solo secondo caso passa cón un ramo per $P$. 


\section{I. La postulazione di una curva multipla.}

7. Denomino (insieme col sig. CAyleY) postulazione di una curva $C$, rispetto ad una superficie $F_{n}$ d'ordine $n$, il numero delle condizioni lineari che $F_{n}$ dee soddisfare, affinchè per essa la curva $C$ sia multipla secondo un numero $i$.

La postulazione di una curva che sia l'intersezione completa di due superficie si puo determinare direttamente. Per una siffatta curva $C$, intersezione completa di due superficie $P, Q$ rispettivamente d'ordine $p, q$, che si tocchino in $k$ punti, l'ordine $m$ e il rango $r$ sono dati dalle formole

$$
m=p q, \quad r+2 k=p q(p+q-2) .
$$

L'equazione di una superficie $F_{n}$, che debba contenere $C$ come curva $i$-pla è della forma

$$
0=F_{n} \equiv A_{0} P^{i}+A_{1} P^{i-1} Q+\cdots+A_{i} Q^{i} ;
$$

infatti, siccome $F_{n}$ passa per la completa intersezione di $P^{i}$ e $Q$, cosi $F_{n}$ deve avere la forma $\left({ }^{*}\right)$

$$
F_{n} \equiv A_{0} P^{i}+Q \cdot F_{n-q},
$$

dove la superficie $F_{n-q}$ contenga $C$ come curva $(i-1)$-pla; perciò $F_{n-q}$ avrà la forma

e così di seguito.

$$
F_{n-q} \equiv A_{1} P^{i-1}+Q \cdot F_{n-21}
$$

S'indichi con

$$
(N-\rho Q)_{i-\rho}
$$

il numero delle costanti assorbite nella superficie $F_{n-\rho q}$ dalla condizione che essa contenga $C$ come curva $(i-\rho)$-pla; e inoltre si scriva per brevità

Dalla formola

$$
\frac{1}{6}(s+1)(s+2)(s+3)=[s] \text {. }
$$

$$
F_{n} \equiv A_{0} P^{i}+F_{n-q} Q
$$

mediante un processo di numerazione già adoperato da $\mathrm{J}_{\mathrm{ACOBI}}\left({ }^{* *}\right)$, si ottiene

$$
\begin{aligned}
N_{i} & =[n]\left\{-[n-i p]+[n-q]-(N-Q)_{i-1}-[n-i p-q]\right\} \\
& =i p q n-\frac{1}{2} i p q(i p+q-4)+(N-Q)_{i-1}
\end{aligned}
$$

(*) Vedi p. es. Math. Annalen, t. 2, p. 314.

(**) G. Crelle, t 15 , p. 285. 
per $n \geqq i p+q-3$; e invece

$$
N_{i}=i p q n-\frac{1}{2} i p q(i p+q-4)-[n-i p-q]+(N-Q)_{i-1}
$$

per $n<i p+q-3$. Poste così le analoghe espressioni di

$$
(N-Q)_{i-1}, \quad(N-2 Q)_{i-2}, \ldots
$$

e avuto riguardo alla

$$
(N-i Q)_{0}=0
$$

si ottiene il risultato seguente.

Sia $p \geqq q$. Nel caso di $n \geqq i p+q-3$, il postulante di $C$ ha il valore

$$
N_{i}=\frac{1}{2} \frac{i(i+1)}{2 \cdot 3} p q\{6 n-(2 i+1)(p+q)+12\} \text {. }
$$

Ma, se

$$
(i-\rho+1) p+\rho q-3>n \leqq(i-\rho) p+(\rho+1) q-3
$$

per ottenere la postulazione di $G$, bisogna all' espressione precedente di $N_{i}$ aggiungere ancora la quantità positiva

$$
-[n-i p-q]-[n-(i-1) p-2 q]-\cdots-[n-(i-\rho+1) p-\rho q],
$$

e la medesima quantita, per $\rho=i$, è da aggiungere quando in generale

$$
n<p+i q-3 \text {. }
$$

L'espressione di $N_{i}$ si puó anche porre sotto la forma

$$
N_{i}=\frac{i(i+1)}{2 \cdot 3}\{3 n-2 i+5\} m-\frac{1}{2} \frac{i(i+1)(2 i+1)}{2 \cdot 3}(r+2 k) .
$$

8. La ricerca della postulazione di una qualsivoglia curva $G$ si ridurrà al caso di un' intersezione completa, completando $C$ in modo da ottenere appunto l'intersezione completa di due superficie.

Se una curva $K$, d'ordine $M$ e di rango $R$, si spezza in due curve $C, C^{\prime}$ che abbiano rispettivamente gli ordini $m, m^{\prime}$, e i ranghi $r, r^{\prime}$, dalla condizione che per tale spezzamento non si altera il numero dei punti doppi apparenti, si ottiene il numero $s$ delle intersezioni delle due curve

$$
s=\frac{1}{2}\left(R-r-r^{\prime}\right),
$$

ed inoltre è $M=m+m^{\prime}$. La curva spezzantesi in $C, C^{\prime}$ ha la stessa postulazione come $K$. 
Sia ora $K$ la completa intersezione di due superficie d'ordine $p, q$. Se $C$ e $C^{\prime}$ hanno inoltre $k, k^{\prime}$ punti doppi effettivi, la postulazione di $K$ sarà (n. $\left.{ }^{\circ} 6\right)$ :

$$
\begin{aligned}
N_{i}^{\prime} & =\frac{1}{6} i(i+1)(3 n-2 i+5) M-\frac{1}{12} i(i+1)(2 i+1)\left(R+2 k+2 k^{\prime}\right) \\
& =\frac{1}{6} i(i+1)(3 n-2 i+5) m-\frac{1}{12} i(i+1)(2 i+1)(r+2 k) \\
& +\frac{1}{6} i(i+1)(3 n-2 i+5) m^{\prime}-\frac{1}{12} i(i+1)(2 i+1)\left(r^{\prime}+2 k^{\prime}\right) \\
& -\frac{1}{6} i(i+1)(2 i+1) s .
\end{aligned}
$$

In questa formola i soli primi due termini dipendono dai numeri $m$ ed $r+2 k$ della curva $C$; mentre gli altri termini, secondo la scelta di $p$ e $q$, possono ancora assumere una serie di valori differenti. Purchè adunque $n$ sia convenientemente grande, - cioè, in primo luogo, così grande che, giusta il $n .{ }^{\circ} 6$, la formola per $N_{i}^{\prime}$ possa sussistere, e poi così grande ancora, che l'ultimo termine di questa formola rappresenti effettivamente la riduzione della postulazione dovuta alle $s$ intersezioni di $G$ e $C^{\prime}$, vale a dire che queste $s$ intersezioni possano introdursi qui come indipendenti fra loro, la quantità

$$
N_{i}=\frac{1}{6} i(i+1)(3 n-2 i+5) m-\frac{1}{12} i(i+1)(2 i+1)(r+2 k)
$$

esprimerà la postulazione per una qualsivoglia $C$. Pare che non si possano assegnare in generale le modificazioni per valori più piccoli di $n$.

9. Trattasi ora di nuovo di determinare la riduzione cui va soggetto la postulazione di un sistema di curve in virtù di punti multipli di questo sistema medesimo. A tale uopo procederò ancora in modo analogo al n. ${ }^{0} 3$.

Sostituisco cioè ai rami del sistema di curve che escono da un punto multiplo di una superficie $F_{n}$ altrettante rette dotate delle corrispondenti moltiplicità. In questo caso la determinazione della riduzione, che è identica con quella del dato sistema di curve, conduce ad un problema piano.

L'equazione della superficie $F_{n}$ sia

$$
0=F_{n} \equiv x_{4}{ }^{n-l} f_{l}+x_{4}{ }^{n-l-1} f_{l+1}+\cdots+f_{n},
$$

dove $f_{\rho}$ è una funzione omogenea d'ordine $\rho$ delle coordinate $x_{1} x_{2} x_{9}$. Se questa superficie dee possedere più rette multiple $G_{1}, G_{2}, \ldots$ uscenti dal punto $l$-plo $P\left(x_{1}=x_{2}=x_{3}=0\right)$, la medesima proprietà dee competere a tutti i coni

$$
f_{l}=0, \quad f_{l+1}=0, \ldots, \quad f_{n}=0,
$$

vale a dire, le curve piane

$$
f_{l}=0, \quad f_{l+1}=0, \ldots, \quad f_{n}=0
$$


debbono avere punti rispettivamente dotati delle stesse moltiplicità nelle intersezioni delle rette $G_{1}, G_{2}, \ldots$, col piano $x_{4}=0$.

Per tal modo, si ha a determinare il numero delle condizioni che sono assorbite da più punti multipli di una curva piana d'ordine $\rho$ : numero il quale, se $\rho$ non oltrepassa un certo limite, dipende da $\rho$ medesimo. La somma di questi numeri, determinati per $f_{l}, \ldots, f_{n}$, più $\frac{1}{6} l(l+1)(l+2)$, è la postulazione del sistema di rette e del punto $P$, rispetto alla superficie $F_{n}$.

10. La postulazione

$$
\frac{1}{2} \sum_{\sigma} i_{\sigma}\left(i_{\sigma}+1\right)
$$

di un sistema di punti $i$-pli, che abbiano una giacitura generale, rispetto ad una curva piana $f_{\rho}$ d'ordine $\rho$, patisce una riduzione tostochè $f_{\rho}$, in virtù dei punti multipli, debba decomporsi in parti, alcune delle quali siano da contarsi più volte. A cagion della supposta generale giacitura di questi punti, $f_{\rho}$ non pud che decomporsi in curve razionali. Per ogni curva razionale che entri come parte $\alpha$-pla della curva $f_{\varrho}$, la riduzione della postulazione ammonta a

$$
-\frac{1}{2} \alpha(\alpha-1) \text {. }
$$

Questo fatto si può riconoscere geometricamente. Infatti, si consideri un fascio di curve razionali; siccome i punti-base, a motivo della razionalita, non formano alcun speciale sistema di punti d'intersezione, cosi questi punti somministrano l'espressione generale per la postulazione rispetto ad un sistema di $a$ curve del fascio. Se ci dev'essere inoltre un punto $\alpha$-plo, cioè se le $\alpha$ curve del fascio debbono coincidere in una determinata, ciò richiede $\alpha$ condizioni soltanto, e la riduzione del numero delle condizioni ammonta a (*)

$$
\frac{1}{2} \alpha(\alpha+1)-\alpha=\frac{1}{2} \alpha(\alpha-1)
$$

(*) Un'interessante conferma di questo teorema è offerta dal teorema delle trasformazioni piane del sig. Cremona (Memorie dell'Accad. di Bologna, serie $2^{a}$, t. $5 ; 1865$ ). Le curve delle reti nei piani $X, Y$ siano d'ordine $n$, le prime con $\alpha_{i}$, le ultime con $\beta_{i}$ punti $i$-pli fissi $(i=1,2, \ldots n-1)$. Allora dalle note equazioni della trasformazione si ricava la

$$
\frac{1}{\overline{2}}\left(n^{9}-1\right)\left(n^{2}+2\right)=\sum_{i}^{\frac{1}{2}} n i(n i+1) \alpha_{i}-\sum_{i}^{\frac{1}{2}} i(i-1) \beta_{i} .
$$

Il primo membro di questa equazione è il numero delle costanti nell' equazione di una curva d'ordine $n^{2}-1$. Tale è quella che nel piano $X$ corrisponde al sistema dei $\beta_{i}$ punti di $Y$; per essa gli $\propto$ punti fondamentali devono essere punti $n \imath$-pli, con che è completamente determinata. E siccome questa curva contiene come parte integrante $i$-pla la curva razionale corrispondente ad un punto fondamentale $i$-plo, così l'equazione precedente $\mathrm{e}$ in accordo col teorema suesposto. 
Come applicazione, darò la postulazione $P$ di tre punti multipli secondo $i_{1}, i_{2}, i_{3}$, rispetto ad una curva $f_{e}$, che possa contenere come parte integrante una retta multipla. Sia $i_{1} \geqq i_{2} \geq i_{3}$. Secondo la grandezza di $\rho$, sono a distinguersi cinque casi :

a) se $\rho \geqq i_{1}+i_{2}+1$, si ha $(\sigma=1,2,3)$

$$
P=\sum_{\sigma}^{1} i_{\sigma}\left(i_{\sigma}+1\right)
$$

B) se $i_{1}+i_{2}-1>\rho \geq i_{1}+i_{3}-1$, si ha

$$
P=\frac{1}{\sigma} \frac{1}{2} i_{\sigma}\left(i_{\sigma}+1\right)-\frac{1}{2}\left(i_{1}+i_{2}-\rho\right)\left(i_{1}+i_{2}-\rho-1\right)
$$

y) se $i_{1}+i_{3}-1>\rho \geqq i_{2}+i_{3}-1$, si ha

$$
P=\sum_{\sigma} \frac{1}{2} i_{\sigma}\left(i_{\sigma}+1\right)-\frac{1}{2}\left(i_{1}+i_{2}-\rho\right)\left(i_{1}+i_{2}-\rho-1\right)-\frac{1}{2}\left(i_{1}+i_{3}-\rho\right)\left(i_{1}+i_{3}-\rho-1\right),
$$

d) se $i_{2}+i_{3}-1>\rho \geqq \frac{1}{2}\left(i_{2}+i_{2}+i_{3}\right)$, si ha

$$
\begin{array}{r}
P=\sum_{\sigma}^{\frac{1}{2}} i_{\sigma}\left(i_{\sigma}+1\right)-\frac{1}{2}\left(i_{1}+i_{2}-\rho\right)\left(i_{1}+i_{2}-\rho-1\right)-\frac{1}{2}\left(i_{1}+i_{3}-\rho\right)\left(i_{1}+i_{3}-\rho-1\right)- \\
-\frac{1}{2}\left(i_{2}+i_{3}-\rho\right)\left(i_{2}+i_{3}-\rho-1\right),
\end{array}
$$

e rimangono in questo caso, ancora

$$
\frac{1}{2}\left(2 \rho-i_{1}-i_{2}-i_{3}+1\right)\left(2 \rho-i_{1}-i_{2}-i_{3}+2\right)
$$

costanti nell' equazione omogenea della curva;

ह) se $\rho<\frac{1}{2}\left(i_{1}+i_{y}+i_{3}\right)$, si ha $P=\frac{1}{2}(\rho+1)(\rho+2)$,

perchè in questo caso non è più possibile alcuna curva $f_{\rho}$.

11. Ora si dedurrà dai n. ${ }^{i} 9$ e 10 la riduzione della postulazione di un sistema di curve, rispetto ad una superficie $F_{n}$, per $\mathrm{i}$ casi ordinari. Dapprima $F_{n}$ possegra una curva $i_{1}$-pla, ed un'altra $i_{2}$-pla $\left(i_{1} \geqq i_{2}\right)$, che si incontrino in un purto $P$; allora $P$ è un punto $i_{1}$-plo della superficie. Sostituendo ai due rami delle curve in $P$ due rette, la postulazione del nuovo sistema sarà

$$
\begin{aligned}
& \frac{1}{6} i_{1}\left(i_{1}+1\right)\left(i_{1}+2\right)+\left(n-i_{1}+1\right)\left\{\frac{1}{2} i_{1}\left(i_{1}+1\right)+\frac{1}{2} i_{2}\left(i_{2}+1\right)\right\}- \\
& \left.-\left\{\frac{1}{2} i_{2}\left(i_{2}-1\right)+\frac{1}{2}\left(i_{2}-1\right)\left(i_{2}-2\right)+\cdots+3+1\right)\right\} \\
& =\frac{1}{6} i_{1}\left(i_{1}+1\right)\left(3 n-2 i_{1}+5\right)+\frac{1}{6} i_{2}\left(i_{2}+1\right)\left(3 n-2 i_{2}+5\right)-\frac{1}{6} i_{2}\left(i_{2}+1\right)\left(3 i_{1}-i_{2}+1\right) \text {, } \\
& \text { supposto } n \geqq i_{1}+i_{2}-2 \text {; donde segue che il termine di riduzione della po- }
\end{aligned}
$$


da un effettivo punto doppio può considerarsi come termine di riduzione doruta ad esso punto) sarà

$$
-\frac{1}{6} i_{2}\left(i_{2}+1\right)\left(3 i_{1}-i_{2}+1\right)
$$

Invece, se $n<i_{1}+i_{2}-2$, la postulazione deve ancora essere diminuita di

$$
\frac{1}{6}\left(i_{1}+i_{2}-n\right)\left(i_{1}+i_{2}-n-1\right)\left(i_{1}+i_{2}-n-2\right),
$$

perchè gli $i_{1}+i_{2}-n-2$ ultimi termini della prima formola del presente n. ${ }^{\circ}$ in tal caso scompajono.

12. Se inoltre la superficie $F_{n}$ contiene un punto $l$-plo $P$, e delle curve $i$-ple $C$ (ordine $m$, rango $r$ ), che passino con $j_{i}$ rami per $P$, supposto che $l$ sia di tal grandezza che il cono osculatore in $P$ ad $F_{n}$ non abbia parti integranti ripetute più volte, la postulazione del sistema $P G$ sarà pel n.$^{\circ} 9$

$$
\begin{gathered}
\frac{1}{6} l(l+1)(l+2)+\sum_{i} \frac{1}{6} i(i+1)\left\{(3 n-2 i+5) m_{i}-\frac{1}{2}(2 i+1) r_{i}\right\}- \\
-\sum_{i} \frac{1}{6} i(i+1)(3 r-2 i+2) j_{i} .
\end{gathered}
$$

13. Per trattare ancora un esempio distinto di un caso nel quale il cono osculatore in $P$ contenga parti coincidenti, suppongo che la superficie $F_{n}$ possegga tre rette concorrenti in $P$, che siano rispettivamente multiple secondo $i_{1}, i_{2}, i_{3}$; e l'ordine della moltiplicità del punto $P$ sia quello che è determinato mediante queste tre linee multiple. Allora sono da distinguersi due casi :

a) $i_{1} \geqq i_{2}+i_{3}$. Il punto $P$ è $i_{1}$-plo per $F_{n}$, ed il numero delle condizioni per questa superficie sarà :

$$
\begin{gathered}
\frac{1}{6} i_{1}\left(i_{1}+1\right)\left(i_{1}+2\right)+\left(n-i_{1}+1\right) \sum_{\sigma} \frac{t}{2} i_{\sigma}\left(i_{\sigma}+1\right)- \\
-\left\{\frac{1}{2} i_{2}\left(i_{2}-1\right)+\frac{1}{2}\left(i_{2}-1\right)\left(i_{2}-2\right)+\cdots+1\right\}-\left\{\frac{1}{2} i_{3}\left(i_{3}-1\right)+\frac{1}{2}\left(i_{3}-1\right)\left(i_{3}-2\right)+\cdots+1\right\}= \\
(*)=\left[i_{1}-1\right]+\left(n-i_{1}+1\right) \sum_{\sigma} \frac{1}{2} i_{\sigma}\left(i_{\sigma}+1\right)-\left[i_{2}-2\right]-\left[i_{3}-2\right]= \\
=\sum_{\sigma} \frac{1}{6} i_{\sigma}\left(i_{\sigma}+1\right)\left(3 n-2 i_{\sigma}+5\right)-\frac{1}{6} i_{2}\left(i_{2}+1\right)\left(3 i_{1}-i_{2}+1\right)- \\
-\frac{1}{6} i_{3}\left(i_{3}+1\right)\left(3 i_{1}-i_{3}+1\right),
\end{gathered}
$$

precisamente come dal $\mathrm{n} .{ }^{\circ} 11$.

(3) $i_{1}<i_{z}+i_{3}$. Qui $P$ dev'essere un punto $l$-plo, dove

$$
l=\frac{1}{2}\left(i_{1}+i_{2}+i_{s}\right), \text { ovvero }=\frac{1}{2}\left(i_{1}+i_{2}+i_{3}+1\right),
$$

(*) Qui si fa uso della notazione del n.0 7 , cioè $[s]=\frac{1}{6}(s+1)(s+2)(s+3)$. 
secondo che $i_{1}+i_{2}+i_{3}$ è pari o dispari. Siccome ora $i_{2}+i_{3} \geq l>i_{1}$, cosi, giovandoci dell' esempio dato nel n. ${ }^{\circ} 10$, avremo pel numero delle condizioni

$$
\begin{gathered}
\frac{1}{6} l(l+1)(l+2)+(n-l+1) \sum_{\sigma} \frac{1}{2} i_{\sigma}\left(i_{\sigma}+1\right)- \\
-\left[i_{2}+i_{3}-l-2\right]-\left[i_{3}+i_{1}-l-2\right]-\left[i_{1}+i_{2}-l-2\right]= \\
=\sum_{\sigma} \frac{1}{6} i_{\sigma}\left(i_{\sigma}+1\right)\left(3 n-2 i_{\sigma}+5\right)-\sum_{\sigma} \frac{1}{6} i_{\sigma}\left(i_{\sigma}+1\right)\left(3 l-2 i_{\sigma}+2\right)+[l-1]- \\
-\left[i_{2}+i_{3}-l-2\right]-\left[i_{3}+i_{1}-l-2\right]-\left[i_{1}+i_{2}-l-2\right] .
\end{gathered}
$$

Se fosse $n<i_{1}+i_{2}-2$, queste formole per la postulazione subirebbero ancora una correzione positiva, che si puo calcolare facilmente, in modo analogo alla chiusa del $n .^{\circ} 11$.

Finalmente osservo ancora che il caso in cui la superficie $F_{n}$ si decomponesse in più parti coincidenti dả luogo a nuove modificazioni nel valore della postulazione, le quali richiederebbero una speciale investigazione.

\section{Il genere di una superficie.}

14. Gli sviluppi del capitolo II ammettono una diretta applicazione alla ricerca del genere $p$ di una superficie $F_{n}$ : numero che rimane invariabile nelle trasformazioni univoche (eindeutige Transformationen). Infatti, il genere $p$ di una superficie $F_{n}$ d'ordine $n$, secondo una definizione data da me nei Mathematische Annalen (t. 2, p. 315), è uguale al numero delle superficie $F_{n-4}$ d'ordine $n-4$, linearmente fra loro indipendenti che si possono far passare $i-1$ volte per ciascuna curva $i$-pla $C_{i}$ di $F_{n}$, ed $l-2$ volte per ogni punto $l$-plo $P_{l}$ della stessa $F_{n}$; ossia

$$
p=\frac{1}{6}(n-1)(n-2)(n-3)-R \text {, }
$$

dove $R$ è la postulazione del sistema composto delle curve $(i-1)$ ple $C_{i}$ e dei punti $(l-2)$ pli $P_{l}$, rispetto ad una superficie $F_{n-4^{*}}$ Qui il valore $R$ è da prendersi nello stesso senso come nel cap. II, cioè con riguardo alle modificazioni ch' esso subisce nel caso in cui $n-4$ non supera un certo limite.

15. $\grave{\mathrm{E}}$ importante che la formola data superiormente per $p$ puó già servire come definizione del genere, cioe di un numero caratteristico, che debba mantenersi invariato nelle trasformazioni univoche, se si calcola la postulazione $R$ senza riguardo alla grandezza di $n-4$, cioè, se per $R$ si adoperano assolutamente le formole generali, che, secondo il cap. II, valgono 
per $n$ abbastanza grande. Quest'osservazione è stata fatta dal sig. CAYLEY (*); e il sig. Zeuthen, in un bel lavoro sulla trasformazione delle superficie (**) ha, sotto ipotesi assai generali e per via puramente geometrica, dimostrata l'invariabilità, nelle trasformazioni univoche, del numero cosi definito. Per $p>0$ le due definizioni conducono allo stesso numero; ma, mentre verbigrazia pei coni la definizione del $n .^{\circ} 14$ dà sempre $p=0$, invece la seconda definizione somministra un numero che è uguale al genere, preso negativamente, di una sezione piana del cono. In seguito io mi serviró dell'ultima definizione, che è più espressiva.

\section{Applicazione alle trasformazioni univoche (birazionali) nello spazio.}

16. Come complemento alle ricerche recentemente fatte dai sig. ${ }^{i}$ CAYLEY e Cremona e da me sulle trasformazioni birazionali nello spazio (***) esporro qui un sistema di formole, alle quali devono soddisfare i numeri relativi alle superficie trasformanti, e le quali si deducono dalle considerazioni svolte nei capitoli I, II, III.

Affinchè un sistema di superficie $\$$ d'ordine $n$, le quali debbano corrispondere univocamente (eindeutig) ai piani dello spazio $X$, possano formare un sistema trasformante nello spazio $Y$ (un sistema omaloidico, secondo l'espressione del sig. Cremona), debbono esse $\phi$ soddisfare a più condizioni:

a) le $\bar{q}$ devono possedere tanti elementi comuni (curve e punti fondamentali) quanti occorrono perchè esse formino una serie triplamente infinita;

b) tre superficie qualsivogliano della serie devono in generale segarsi in un solo punto variabile;

c) le $\phi$ sono di genere $p=0$, conformemente alla definizione del $n .^{\circ} 15$.

Le formole per le prime due condizioni sono già state date (l. c.) dal sig. Carley, sebbene in una forma un po'meno generale di quella che qui si verrà proponendo. Le formole risultanti dalle tre condizioni costituiscono un sistema chiuso, che è l'analogo di quello che il sig. Cremona ha stabilito per le trasformazioni piane.

(*) Math. Annalen, t. 3, pag. 526.

(**) Math. Annalen, t. 4, pag. 42 [III].

(***) Veggasi la gia citala Memoria di CAvLey, e inoltre i lavori pubblicati contemporaneamente da Cremona e da me, il primo nelle Götting. Nachrichten $1871, \mathbf{n} .{ }^{\circ} \mathbf{5}$, il mio nei Math. Annalen t. 3; come pure le ricerche ancor più generali di Cremona nei Rendiconti dell' Istituto Lombardo, 4 maggio e 1 gingno 1871 , e nel presente fascicolo degli Annali di Matematica. 
In ciò che segue, io non tratterò che un caso generale. Le modificazioni delle formole nei easi speciali si dovranno dedurre per la via indicata superiormente.

17. Le superficie $\phi$ d'ordine $n$ abbiano in comune le curve fondamentali $i$-ple $C_{i}$ d'ordine $m_{i}$ e di rango $r_{i}$; una curva $C_{i}$ abbia $k_{i i}$ punti doppi effettivi, e incontri in altri $k_{i j}$ punti una curva $C_{j}(i \geqq j)$. Oltracció, le $\phi$ abbiano ancora i punti fondamentali $l$-pli $P_{l}$, pei quali una curva $C_{i}$ passi con $j_{i l}$ rami. Qui però io suppongo che il cono osculatore in un cosi fatto punto $P_{\imath}$ non sia fisso, nè tutto nè in parte, per tutte le $\phi$. Da ultimo le $\phi$ abbiano ancora dei punti di contatto dell'ordine $\sigma-1$.

La condizione $b$ ) nel $n .^{\circ} 16$ dà, giusta il cap. I, la seguente equazione d'equivalenza:

$$
\left\{\begin{array} { c } 
{ n ^ { 3 } - 1 = \sum _ { i } i ^ { 2 } \{ ( 3 n - 2 i ) m _ { i } - i r _ { i } \} - \sum _ { i j } i ^ { 2 } ( 3 j - i ) k _ { i j } + } \\
{ + \sum _ { i } l ^ { 3 } - \sum _ { i l } i ^ { 2 } ( 3 l - 2 i ) j _ { i l } + \sum _ { \sigma } \sigma ^ { 2 } }
\end{array} \quad \text { per } \left\{\begin{array}{l}
i \equiv j \\
i \equiv l
\end{array}\right.\right.
$$

La prima somma doppia $\sum_{i j}$ dev'essere estesa a tutt' i punti d'intersezione delle curre fondamentali prese a due a due, eccettuati quelli che cadono nei $P_{l}$, e a tutt' i punti doppi effettivi di ciascuna curva $C_{i}$. La seconda somma doppia $\sum_{i}$ dev'essere estesa dapprima a tutt'i rami delle curve $C_{i}$ passanti per un punto $P_{l}$, indi a tutt' i diversi punti $P_{l}$.

In grazie del cap. II, la condizione a) dà la seguente equazione di postulazione:

$$
\left\{\begin{array}{c}
\frac{1}{6}(n+1)(n+2)(n+3)-4=\sum_{i} \frac{1}{6} i(i+1)\left\{(3 n-2 i+5) m_{i}-\frac{1}{2}(2 i+1) r_{i}\right\}- \\
-\sum_{i j} \frac{1}{6} i(i+1)(3 j-i+1) k_{i j}+\sum_{l} \frac{1}{6} l(l+1)(l+2)- \\
-\sum_{i l} \frac{1}{6} i(i+1)(3 l-2 i+2) j_{i l}+\sum_{\sigma} \frac{1}{2} \sigma(\sigma+1)
\end{array}\right.
$$

e finalmente, pel cap. III, $\mathrm{n}^{\circ}$ 15, la condizione $c$ ) dà l'equazione del genere:

$$
\left\{\begin{aligned}
& \frac{1}{6}(n-1)(n-2)(n-3)=\sum_{i} \frac{1}{6} i(i-1)\left\{(3 n-2 i-5) m_{i}-\frac{1}{4}(2 i-1) r_{i}\right\}- \\
&- \sum_{i j} \frac{1}{6} i(i-1)(3 j-i-1) k_{i j}+\sum_{i} \frac{1}{6} l(l-1)(l-2)- \\
&-\sum_{i l} \frac{1}{6} i(i-1)(3 l-2 i-2) j_{i l}
\end{aligned}\right.
$$


Da queste tre equazioni, con facile combinazione, si ricavano le seguenti:

$$
\left\{\begin{array}{c}
\frac{1}{6}(4 n-3)(4 n-2)(4 n-1)-1=\sum_{i} \frac{1}{6} 4 i(4 i-1)\left\{(12 n-8 i-5) m_{i}-\frac{1}{2}(8 i-1) r_{i}\right\}- \\
-\sum_{i j} \frac{1}{6} 4 i(4 i-1)(12 j-4 i-1) k_{i j}+\sum_{l} \frac{1}{6}(4 l-2)(4 l-1) 4 l- \\
-\sum_{i l} 4 i(4 i-1)(12 l-8 i-2) j_{i l}+\sum_{\sigma} \sigma(7 \sigma-3),
\end{array}\right.
$$

$$
\begin{aligned}
2\left(n^{2}-1\right) & =\sum_{i} i\left\{(n+i) m_{i}-\frac{1}{2} i r_{i}\right\}-\sum_{i j} i j k_{i j}+ \\
& +\sum_{l} l^{2}-\sum_{i l} i l j_{i l}+\sum_{\sigma} \frac{1}{2} \sigma(\sigma+1),
\end{aligned}
$$

(6) $11(n-1)=\sum_{i} i\left(5 m_{i}-\frac{1}{2} r_{i}\right)-\sum_{i j} i k_{i j}+\sum_{i} 2 l-\sum_{i l} 2 i j_{i l}+\sum_{\sigma} \frac{1}{2} \sigma(\sigma+3)$.

Affine di completare questo sistema di formole per le trasformazioni razionali nello spazio, si designino le analoghe quantità relative allo spazio $X$, colle stesse notazioni, come per lo spazio $Y$, ma coll'aggiunta di un accento. Allora per gli ordini $n, n^{\prime}$ delle superficie trasformanti $\phi, \psi$ degli spazi $Y$, $X$ risp., si ha :

$$
n^{\prime}=n^{2}-\sum_{i} i^{2} m_{i}, \quad n=n^{\prime s}-\sum_{i} i^{2} m_{i}^{\prime},
$$

e a cagione dell'uguaglianza del genere delle sezioni piane delle superficie trasformanti nei due spazi,

$$
\frac{1}{2}(n-1)(n-2)-\sum_{i} \frac{1}{2} i(i-1) m_{i}=\frac{1}{2}\left(n^{\prime}-1\right)\left(n^{\prime}-2\right)-\sum_{i} \frac{1}{2} i(i-1) m^{\prime}{ }^{\prime}
$$

donde

$$
4\left(n^{\prime}-n\right)=\sum_{i} i m_{i}^{\prime}-\sum_{i} i m_{i}
$$

18. Le due equazioni (4) e (5) si possono interpretare geometricamente. La (4) dice che la Jacobiana delle $\phi$, superficie d'ordine $4 n-4$, dotata di un punto $(4 l-2)$ plo $P_{l}$ e di una curva $(4 i-1)$ pla $C_{i}$, è completamente determinata da queste condizioni; essa (4) è adunque l'equazione di postulazione per la Jacobiana delle $\phi$. E merita d'essere notato che, in questo caso, le espressioni date nel cap. II pel postulante possono essere adoperate senza modificazioni, sebbene la Jacobiana in generale contenga delle parti multiple (coincidenti), quali sono le superficie che corrispondono ai punti fondamentali di $X$.

Invece della (5), considero l'equazione che col mezzo della

$$
n^{\prime}=n^{2}-\Sigma i^{2} m
$$


se ne ricava, cioè

$(5)^{\prime}\left\{\begin{aligned} 4 n^{\prime}-4 & =\sum_{i} 2 i\left\{(n-i) m_{i}-\frac{1}{2} i r_{i}\right\}-\sum_{i j} 2 i j k_{i j}+ \\ & +\sum_{i} 2 l^{2}-\sum_{i l} 2 i l j_{i l}+\sum_{\sigma}^{\mathbf{\Sigma}} \sigma(\sigma+1) .\end{aligned}\right.$

Le curve razionali $S$, intersezioni variabili delle superficie $\phi$ prese a due a due, sono d'ordine $n^{\prime}$. Il secondo membro della (5)' esprime il numero $4 n^{\prime}-4$ delle condizioni alle quali debbono soggiacere queste curve $S$, mentre esše hanno in comune colla $C_{i}$ il numero di punti dato nei n. ${ }^{\mathrm{i}} 4$ e 5 , e passano per $P_{l}$ col numero di rami ivi del pari assegnato. Infatti, una curva $S$ incontra una curva $C_{i}$ in

$$
\begin{gathered}
Q_{i}=2 i\left\{(n-i) m_{i}-\frac{1}{2} i r_{i}\right\}-\sum_{h} h^{2} k_{h i}-\sum_{j}\left(2 i j-i^{2}\right) k_{i j}-\sum_{i}\left(2 i l-2 i^{2}\right) j_{i l} \\
(h<i, \quad j \geqq i)
\end{gathered}
$$

punti (dove, per un punto doppio effettivo di $C_{i}$ la somma $\sum_{j}$ deve estendersi ad entrambi i rami del nodo); passa inoltre con

$$
R_{l}=l^{2}-\sum_{i} i^{2} j_{i l}
$$

rami per $P_{l}$, ed ha in un punto di contatto d'ordine $\sigma-1$, delle $\phi$, un punto $\sigma$-plo assorbente $\sigma(\sigma+1)$ condizioni.

Inoltre $4 n^{\prime}-4$ è anche l'ordine della Jacobiana delle superficie trasformanti $\psi$ dello spazio $X$; la formola $(5)^{\prime}$ dà dunque l'ordine $Q_{i}$ della superficie che in $X$ corrisponde ad una curva $C_{i}$, e l'ordine $R_{l}$ della superficie corrispondente ad un punto $P_{l}$ : la quale ultima è contenuta due volte nella Jacobiana delle $\psi$. Dall'ultimo termine della formola $(5)^{\prime}$ si conclude ancora che la superficie d'ordine $\sigma$ corrispondente ad un punto di contatto, d'ordine $\sigma-1$, delle $\phi$ è contenuta $\sigma+1$ volte nella Jacobiana anzidetta $(*)$.

Heidelberg, settembre 1871 .

(*) Questa conclusione in modo analogo è già stata ottenuta dal sig. Cremona nella $2^{a}$ delle gia citate Note dei Rend. Ist. Lomb. 